\title{
Optimization of cultivation conditions of Salvia viridis L. shoots in the Plantform bioreactor to increase polyphenol production
}

\author{
Izabela Grzegorczyk-Karolak ${ }^{1}$ (D) $\cdot$ Paulina Staniewska ${ }^{1}$ (D) $\cdot$ Liwia Lebelt $^{2}$ (D) $\cdot$ Dorota G. Piotrowska $^{2}$ (D)
}

Received: 12 July 2021 / Accepted: 3 September 2021 / Published online: 13 September 2021

(c) The Author(s) 2021

\begin{abstract}
Salvia viridis is a rich source of pharmacologically-important polyphenolic compounds with anti-inflammatory, antioxidant and anticancer activities. The aim of the present study was to optimise the large-scale cultivation of sage shoots in the Plantform bioreactor. It evaluated the effect of culture duration (two or three weeks), the use of a semi-continuous system and the immersion frequency in a liquid medium (every 80 or $170 \mathrm{~min}$ ) on the growth and accumulation of secondary metabolites. The content of bioactive phenolic acids and phenylethanoids in the obtained material was determined using HPLC. The most efficient growth parameters, expressed as fresh $(26.99 \mathrm{~g})$ and dry weight $(3.51 \mathrm{~g})$ as well as proliferation ratio (18.6), were observed for shoots grown in a fed-batch system, immersed every $80 \mathrm{~min}$; this approach yielded a 33-fold biomass increase within four weeks. However, the most efficient production of bioactive compounds was achieved for shoots grown for three weeks in a batch system, immersed every $80 \mathrm{~min}$ : total phenolic acid, total phenylethanoid and total phenol contents of 18.3, 11.4 and $29.7 \mathrm{mg} / \mathrm{g}$ DW. In these conditions, within three weeks, total phenolic acid level was almost 10 times higher than that found in the aerial parts of four-month-old soil-grown plants with a similar phenylethanoid level. Hence, the described $S$. viridis shoot culture cultivated in the Plantform bioreactor may be an alternative efficient source of plant material rich in valuable compounds.
\end{abstract}

\section{Key message}

Optimization of culture conditions (culture duration, the use of fed-batch mode, frequency of the medium application) for growth and productivity of bioactive compounds in Salvia viridis shoots cultivated in the Plantform bioreactor.

Keywords Immersion frequency $\cdot$ Phenylethanoids · Polyphenolic acid $\cdot$ Salvia viridis $\cdot$ Shoot culture $\cdot$ Temporary immersion system

$\begin{array}{ll}\text { Abbreviations } \\ \text { MS } & \text { Murashige \& Skoog medium } \\ \text { IAA } & \text { Indole-3-acetic acid } \\ \text { m-Top } & \text { Meta-topolin } \\ \text { DW } & \text { Dry weight } \\ \text { FW } & \text { Fresh weight }\end{array}$

Communicated by Ali R. Alan.

Izabela Grzegorczyk-Karolak

izabela.grzegorczyk@umed.lodz.pl

1 Department of Biology and Pharmaceutical Botany, Faculty of Pharmacy, Medical University of Lodz, Muszynskiego 1, 90-151 Lodz, Poland

2 Bioorganic Chemistry Laboratory, Faculty of Pharmacy, Medical University of Lodz, Muszynskiego 1, 90-151 Lodz, Poland
TIS Temporary immersion system

HPLC High-performance liquid chromatography

RA Rosmarinic acid

VB Verbascoside

MRA Methyl rosmarinate

MART Martynoside

LEU Leucosceptoside A

ISOVB Isoverbascoside

CA Caffeic acid

CAH Caffeic acid hexoside

SAF(I) Salvianolic acid F isomer (I)

SAF(II) Salvianolic acid F isomer (II)

SAB Salvianolic acid B 


\section{Introduction}

Salvia viridis L. (syn. Salvia horminum L.), also commonly-known as red-topped sage, painted sage, annual clary and Joseph's sage, is an annual or biennial member of the Lamiaceae family (Bown 2002). Being native to the Mediterranean countries and Anatolia (Turkey), it prefers dry, rocky areas as well as fields, wastelands and dunes. It can also be found in Iran and in the Caucasus (Davis 1982) where it has long been known for its medicinal properties in traditional medicine. For centuries, it has been used as a remedy for infectious diseases and inflammatory disorders, especially affecting the eyes and oral cavity (Dweck 2000; Ghorbani 2005).

$S$. viridis is rich in biologically-active chemical compounds, most of which are polyphenols and terpenoids; however, mono- and sesquiterpene hydrocarbons are also present in the essential oil (Yayli et al. 2010). The aerial parts are mainly rich in polyphenolic derivatives such as phenolic acids, phenylethanoids and flavonoids, with rosmarinic acid, verbascoside and glycoside derivatives of apigenin and luteolin dominating (Grzegorczyk-Karolak and Kiss 2018). In the roots of the species, polyphenolic acids and diterpene derivatives predominate with small amounts of flavonoids and phenylethanoids also being present (Rungsimakan 2014; Zengin et al. 2019).

While $S$. viridis is mainly cultivated as an ornamental plant in Europe, increasing attention is being focused on the multidirectional pharmacological potential of its shoots and roots. Rosmarinic acid and verbascoside demonstrate rich and well-documented biological activity, and are present in significant amounts in extracts from $S$. viridis. The varied chemical composition of the plant makes it potentially useful in disorders such as diabetes (Naimi et al. 2017; Wu et al. 2020), Alzheimer's (Habtemariam 2018) and Parkinson's diseases (Lv et al. 2019) and depression (Liang et al. 2016; López-Rodríguez et al. 2019). It may also be used to treat inflammatory disorders (Lee et al. 2013; Alipieva et al. 2014; Luo et al. 2020), as well as these caused by bacteria such as Staphylococcus aureus, Bacillus cereus, Pseudomonas aeruginosa, Escherichia coli or Proteus vulgaris (Ulubelen 2003; GrzegorczykKarolak et al. 2019) and fungi such as Candida spp. and Aspergillus brasiliensis (Grzegorczyk-Karolak et al. 2019). Compounds isolated from the plant also show antiviral activity; they have been found to inhibit Herpes simplex virus type 1 attachment to host cells and affect their penetration, inhibit HIV-1 integrase, an enzyme essential for viral replication, and reduce influenza-induced lethality and clinical syndromes in mice in vivo (Tewtrakul et al. 2003; Astani et al. 2014; Hu et al. 2016). They also show effective action against several species of protozoa, e.g.
Leishmania donovani, L. amazonensis, or Trypanosoma brucei rhodesiense (Wu et al. 2020). Thanks to its antioxidant activity, the species is a valued agent in the cosmetic and food industries (Grzegorczyk-Karolak and Kiss 2018). $S$. viridis extracts have also been found to have anticancer (Nadeem et al. 2019; Wu et al. 2020) and anti-angiogenic (Vieira et al. 2020) properties. Other biological activities of this plant include: photo- (Alipieva et al. 2014), gastro- (López-Rodríguez et al. 2019), cardio- (Ulubelen 2003; Quan et al. 2021), hepatoprotective (Elufioye and Habtemariam 2019) and even immunosuppressive (Yun et al. 2003) effects.

As the plant demonstrates such broad therapeutic potential, including civilization diseases, and considering that it is an annual plant, requiring annual sowing, there is a clear need to develop effective methods for its cultivation. Due to the almost unlimited demand for high-quality standardized sources of bioactive compounds, which regrettably results in the extinction of species, alternative methods of meeting them, such as in vitro plant cultures, are being investigated.

Plant biotechnology allows fast, efficient and easily reproducible production of plant raw materials and their isolated compounds. In vitro cultures offer many advantages over traditional plant cultivation methods, such as independence from climatic and geographical conditions, possibility of efficient propagation of species that are difficult to reproduce in vivo or obtaining large amounts of raw material in a short time from a small quantity of explants (Sharma et al. 2015; Espinosa-Leal et al. 2018). However, an important step in the optimization of cultivation, necessary for its profitability, is increasing its scale; one approach to this is through the use of special bioreactors for the propagation of plants and their organs.

Bioreactor cultivation allows manual operations to be streamlined and automated, saves time and reduces risk of infection, thus reducing costs of procedure. It also offers the possibility of monitoring and adjusting culture conditions at different stages. Additionally, the use of liquid media gives explants better access to nutrients evenly dispersed in the medium, which often increases yield. Moreover, unlike solid media, the nutrients are not absorbed by gelling agents, whose removal from the media results in additional reduction of culture costs (Tripathi and Tripathi 2005).

Recent reports indicate that temporary immersion systems (TIS) seem to be particularly beneficial for plant breeding. They allow to avoid complete and continuous immersion of plant organs in the medium, which often results in various types of anomalies and growth disorders, including hyperhydricity (Damiano et al. 2005). An example of such a system is the Plantform bioreactor (Plant Form, Sweden). So far, it has been successfully used for the propagation of a some of medical plants, including Olea europaea L. (Benelli and De Carlo 2018), Phoenix dactylifera L. (Almusawi et al. 
2017), Rubus idaeus L., Echinacea purpurea (L.) Moench. (Welander et al. 2014), Vaccinium corymbosum L., V. angustifolium L. (Welander et al. 2017) or Schisandra chinensis (Turch.) Baill. (Szopa et al. 2017). However, no studies appear to have examined the in vitro cultivation of Salvia spp., including $S$. viridis, in this type of bioreactor.

Therefore, the aim of this study was to establish a culture of Salvia viridis L. shoots in the Plantform bioreactor and evaluate the level of bioactive phenols in the obtained plant material. Previous biotechnological studies on $S$. viridis have focused on differentiated and undifferentiated cultures of this species (Grzegorczyk-Karolak et al. 2019), as well as on the optimization of the conditions for their growth and bioactive metabolite production (Grzegorczyk-Karolak et al. 2020). Other studies have examined the genetic transformation of $S$. viridis by Agrobacterium rhizogenes and obtaining hairy root cultures which, after their optimization, produced 10-times higher levels of polyphenolic acids compared to the roots of naturally-grown plants (Grzegorczyk-Karolak et al. 2018; Grzegorczyk-Karolak 2020).

Since the growth of cultures in a bioreactor system and their secondary metabolite accumulation could strongly depend on the cultivation conditions, the present study examines the effect of changes in culture duration (i.e. two or three weeks) and the frequency of immersing the culture in the medium (i.e. every 80 or $170 \mathrm{~min}$ ). The effect of modifying the culture by adding a fresh portion of the medium after two weeks (fed-batch system) was also tested.

\section{Materials and methods}

\section{Plant material}

The explants used in the study were obtained from S. viridis shoot culture maintained as described earlier (Grzegorczyk-Karolak et al. 2019) and grown on MS (Murashige and Skoog 1962) solid (0.7\% agar) medium with $0.1 \mathrm{mg} / \mathrm{L}$ IAA and $1 \mathrm{mg} / \mathrm{L} \mathrm{m}$-Top. This growth regulator combination has been experimentally established as optimal during previous research (Grzegorczyk-Karolak et al. 2020).

\section{Shoot culture in the Plantform bioreactor}

Shoots were cultivated in a bioreactor with the Plantform temporary immersion system (PlantForm, Hjärup, Sweden). The bioreactor consists of a transparent polycarbonate container, a frame with four legs and a polypropylene chamber. Air flow is controlled through ports equipped with $0.2 \mu \mathrm{m}$ filters, two of which are connected through polypropylene sterilized filters to the system that pumps sterile air into the lower chamber of the bioreactor, which contains the medium. The chamber includes a basket with holes, on which the plant material is placed. The apparatus is closed from the top with a snap polypropylene lid, isolated with silicon ring from the inside. The aeration and immersion period of the culture was regulated using two Hailea air pumps (Guangdong Hailea Group Co., Ltd, Guangdong, China): ACO-9610 (power $10 \mathrm{~W}$; capacity $10 \mathrm{~L} / \mathrm{min}$ ) and ACO-9602 (power $5 \mathrm{~W}$; capacity $7.2 \mathrm{~L} / \mathrm{min}$ ) connected with bioreactor via silicon tubes. Immersion time and frequency were programmed with a timer (ESIC AX300) connected to the device. More detailed information about the bioreactor system is available on the manufacturer's website. Before use, the bioreactor and the medium were sterilized at a temperature of $121{ }^{\circ} \mathrm{C}$, under a pressure of $1 \mathrm{~atm}$., for $17 \mathrm{~min}$.

For cultivation, about $0.84 \mathrm{~g} \mathrm{FW}(0.11 \mathrm{~g} \mathrm{DW})$ inoculum, comprising 18 shoots containing the apical meristem approximately $1 \mathrm{~cm}$ in length, was placed in Plantform bioreactor (Fig. 1A). Initially, the bioreactor contained $500 \mathrm{~mL}$ of MS medium with $0.1 \mathrm{mg} / \mathrm{L}$ IAA and $1 \mathrm{mg} / \mathrm{L}$ m-Top. The explants was immersed with the medium every $80 \mathrm{~min}$ for $10 \mathrm{~min}$. The cultures were grown in batch mode, and the shoot growth parameters were estimated after 2 and 3 weeks.

The next stage of the experiment tested the fed-batch culture system. Briefly, the bioreactor initially contained $450 \mathrm{~mL}$ of the growth medium; 2 weeks after inoculation, this amount was supplemented with $300 \mathrm{~mL}$ fresh medium, making a total of $750 \mathrm{~mL}$. In such cases, the growth parameters were determined after a further week and two weeks: the total duration of the culture was 3 and 4 weeks.

In the last stage of the experiment, the number of daily immersion periods was halved (the culture was immersed every $170 \mathrm{~min}$ for $10 \mathrm{~min}$ ). In this experiment, the culture was kept under earlier established optimal growth conditions; after two weeks the medium was partially refilled, and the growth parameters were determined four weeks after inoculation.

All cultures were grown in a growth chamber at $26 \pm 2{ }^{\circ} \mathrm{C}$, $80-90 \%$ humidity, under a $16 \mathrm{~h}$ photoperiod in artificial light (fluorescent lamps; $40 \mu \mathrm{m} \mathrm{m}^{-2} \mathrm{~s}^{-1}$ ). After a cultivation time appropriate to the treatment, the mean number of buds/shoots formed per explant (proliferation ratio), the mean shoot length and the ratio of obtained shoots to buds (structures less than $0.5 \mathrm{~cm}$ long) were determined. Shoot morphology and fresh (FW) and dry weight (DW) of culture [g/culture] were also established.

\section{Phytochemical analysis}

Following cultivation, shoots from all treatments were collected for analysis. The plant material $(100 \mathrm{mg}$ ) was lyophilised, pulverized, extracted three times using methanol/ water (8:2 v/v) as described earlier (Grzegorczyk-Karolak and Kiss 2018), and combined extracts were evaporated under reduced pressure. 

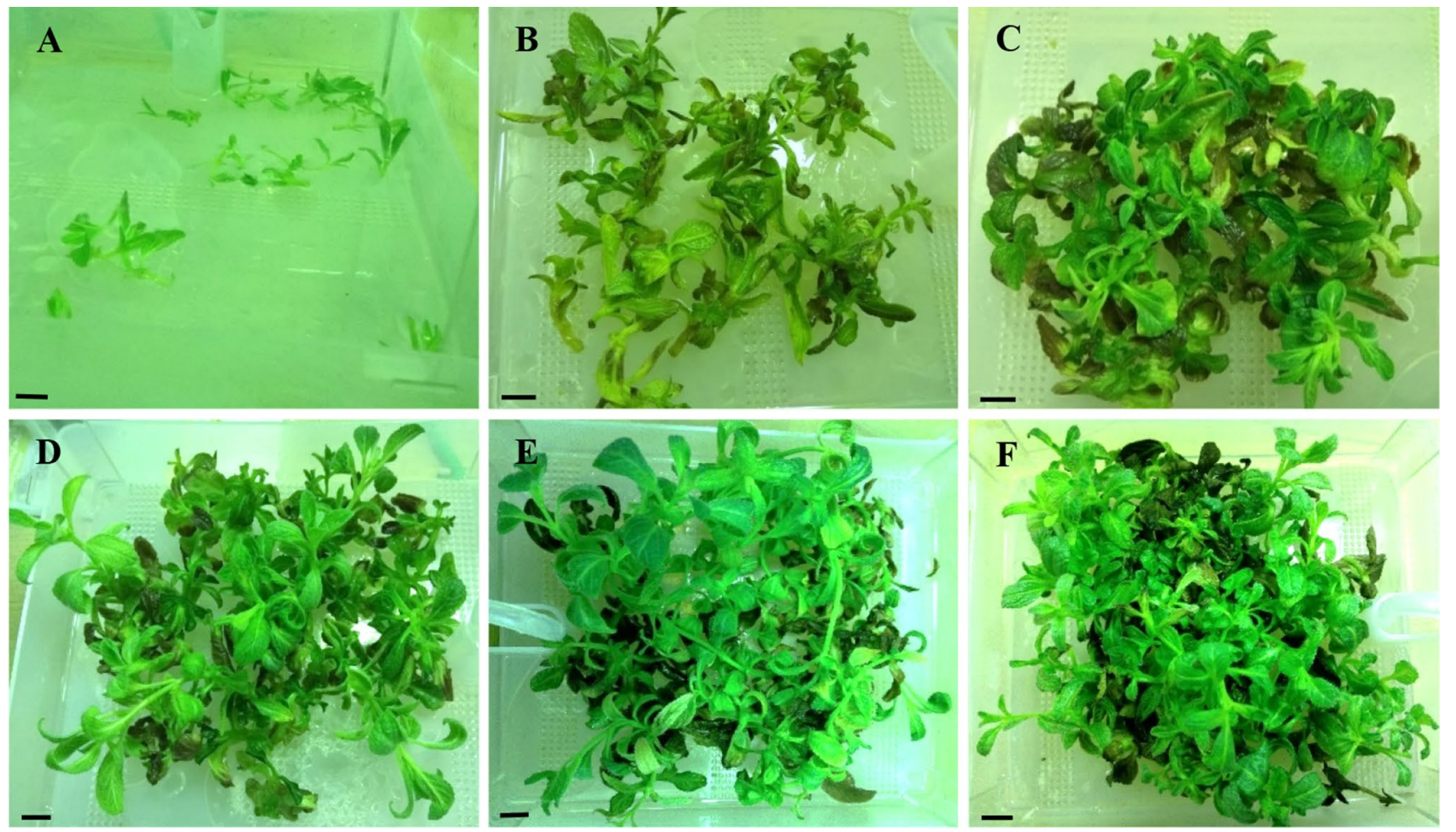

Fig. 1 A Inoculate: 5-week-old shoot tips of S. viridis placed in the Plantform bioreactor. $S$. viridis shoots cultured in the Plantform bioreactor in MS liquid medium with $0.1 \mathrm{mg} / \mathrm{L}$ IAA and $1 \mathrm{mg} / \mathrm{L} \mathrm{m}$-Top, at immersion frequency every $80 \mathrm{~min}$ : B after 2 weeks of batch culture,
C after 3 weeks of batch culture, D after 3 weeks of fed-batch culture, $\mathbf{E}$ after 4 weeks of fed-batch culture; at immersion frequency every $170 \mathrm{~min} \mathbf{F}$ after 4 weeks of fed-batch culture. Bar $1 \mathrm{~cm}$
The dry extract was dissolved in $2 \mathrm{~mL}$ methanol:water solution $(8: 2 \mathrm{v} / \mathrm{v})$. Quantitative analysis was carried out using a Waters apparatus consisting of a Waters 2545 binary gradient module pump, Waters 2767 auto sampler and Waters 2998 diode array detector. Separation was performed on a XBridge C18 OBD column $(4.6 \times 100 \mathrm{~mm})$ with a particle size of $5 \mu \mathrm{m} .0 .1 \%$ trifluoroacetic acid in water (A) and $0.1 \%$ trifluoroacetic acid in acetonitrile (B) were used as a chromatographic eluent. The gradient elution used for analysis was follows: $0-20 \mathrm{~min} 5-50 \%$ solvent $\mathrm{B}, 20-21 \mathrm{~min}$ $50 \%$ solvent B, 21-22 min 50-5\% solvent B, 22-27 min 5\% solvent $\mathrm{B}$. The flow rate was $1.6 \mathrm{~mL} / \mathrm{min}$, and the injection volume was $4 \mu \mathrm{L}$. UV spectra were recorded over range of 190-700 nm, chromatograms for quantitative analysis were acquired at $325 \mathrm{~nm}$. The compounds were identified by a comparison of their retention times and MS and UV spectra with those of standard compounds and/or literature data as described previously (Grzegorczyk-Karolak and Kiss 2018; Grzegorczyk-Karolak et al. 2019).

The compounds were analysed quantitatively by comparing the peak areas of standards and analysed samples. Individual standard calibration curves were constructed based on the area peaks constructed in the concentration range $1-1000 \mu \mathrm{g} / \mathrm{mL}$ for RA, $25-400 \mu \mathrm{g} / \mathrm{mL}$ for $\mathrm{VB}, 1-100 \mu \mathrm{g} /$
$\mathrm{mL}$ for $\mathrm{SAB}$ and $\mathrm{CA}$. Pure reference standards of CA, RA, $\mathrm{SAB}$ were purchased from Sigma Aldrich (Darmstadt, Germany) and VB- from Phytoplan (Heidelberg, Germany). Any compounds for which pure standards were not available were quantified according to the calibration curve of similar standards as described earlier (Grzegorczyk-Karolak et al. 2019). The regression equations were: for VB $\mathrm{y}=0.021 \mathrm{x}-3.9767$ $\left(R^{2}=0.993\right)$, for CA $y=0.0056 x+0.021\left(R^{2}=0.9992\right)$, for RA $y=0.008 x+14.744\left(R^{2}=0.997\right)$, and for $S A B$ $y=0.0227 x+5.5702\left(R^{2}=0.9805\right)$. The total content of different chemical groups, calculated as total phenylethanoid, total phenolic acid and total phenol content, was calculated by summing up the contents of individual quantified compounds. The concentration of the compounds in the analysed samples was presented in $\mathrm{mg} / \mathrm{g}$ DW.

\section{Statistical analysis}

The experiments were performed in triplicate. The results are presented as mean value \pm standard error (SE). All data were calculated using MS-Excel 2013 (Microsoft Sp. z o. o., Warsaw, Poland). STATISTICA 10 software (STATSoft, Krakow, Poland) was used for statistical analysis. Differences between means were tested for statistical significance 
with the one-way ANOVA test and multivariate analysis of variance, followed by the Tukey's test. Values marked with the same letter were not significantly different (at $p \leq 0.05$ ).

\section{Results}

\section{Propagation and biomass growth}

The effect of different cultivation variants on S. viridis culture growth and production of secondary metabolites was evaluated. In all protocols, the shoots cultivated in Platform bioreactor showed vigorous growth and normal morphology, regardless of treatment.

Initial observations concerned the optimal time for cultivating shoots in a bioreactor with a temporary immersion system. Most explants from the batch culture immersed in the medium 10 min every 80 min demonstrated shoot and/or bud formation after two weeks (Fig. 1B). After this cultivation time, the proliferation ratio was 8.2 (Fig. 2A), and the fresh weight of the culture reached $9.1 \mathrm{~g}$ (Fig. 2C), while the dry weight was $1.38 \mathrm{~g}$ (Fig. 2D). Extending the duration of the culture by another week resulted in intense growth of the culture (Fig. 1C). Although the proliferation index increased only slightly during third week of batch culture (8.9), and the mean length of the main and axillary shoots did not change significantly (Fig. 2E-F), the biomass of the culture doubled during this time. A significant change was also observed in the percentage ratio of obtained shoots to buds (from 23 to $37 \%$ ) (Fig. 2B). However, at the end of the third week of culture, the shoots, especially their lower leaves, started to darken, which could indicate aging of the culture due to depletion of nutrients in the medium. Further extending the duration of culture in the batch system proved impossible.

The next stage of the experiment was based on a semicontinuous system with supplementation with extra medium two weeks after initial inoculation. The immersion frequency of $S$. viridis shoots in the medium was not changed. Evaluation of growth parameters three weeks after initiation of culture (Fig. 1D) revealed 30\% higher fresh and 20\% higher dry weight (Fig. 2C-D) than that obtained at the same time without medium supplementation. The changes in biomass were associated with a statistically significant increase in the proliferation ratio (13.4), a significant increase in the length of the main shoots and a significantly higher ratio of shoots to buds formed on the explants (Fig. 2B). After supplementation, the culture demonstrated active growth for three weeks and the shoots showed no changes in morphology or necrosis. This was decisive in extending the duration of the fed-batch culture by another week, up to four weeks. After another week, only a slight increase in biomass was observed; however, a further increase in the proliferation ratio and elongation of shoots, especially the main shoots, was observed. These two parameters reached their optimal values in this cultivation treatment: proliferation ratio of 18.6 and elongation above $10 \mathrm{~cm}$ (Fig. 2). At the end of this period, however, aging and shoot darkening were observed (Fig. 1E).

The last stage of the research examined the effect of modifying the immersion frequency of the shoots in the fedbatch cultivation variant optimized for culture growth. In this variant, the shoots were again immersed in the medium for a period of $10 \mathrm{~min}$, but at half the frequency, i.e. every 170 min (Fig. 1F). The S. viridis shoot culture immersed at this new frequency demonstrate slightly higher DW and FW, compared to those immersed every 80 min (Fig. 2CD). They also demonstrated a higher ratio of axillary shoots to buds, i.e. from 38 to $51 \%$ (Fig. 2B), and greater axillary shoot length, i.e. about 1.2 times (Fig. 2F). On the other hand, fewer shoots/buds developed for the new variant (Fig. 2A), and the main shoots were significantly shorter (Fig. 2E). It was also observed that the leaves of shoots immersed every 170 min started to darken earlier, but the process was slower and less intensive in comparison with culture immersed twice as often.

\section{Phenolic compound production}

Six phenolic acids and four phenylethanoids were identified in the S. viridis shoot extract using UPLC-PDA-ESI-MS/ MS, as described earlier (Grzegorczyk-Karolak et al. 2020). The same compounds were revealed in all extracts taken from all bioreactor cultivation variants; however, quantitative differences were revealed by HPLC analysis (Fig. 3). The content of individual compounds is presented in Table 1. In all samples, the predominant phenolic acid was rosmarinic acid (RA), and the predominant phenylethanoid was verbascoside (VB); the level of RA was higher than VB.

Longer cultivation time, regardless of whether the batch or fed-batch system was used, favored higher levels of most bioactive compounds. However, while the fed-batch system, i.e. where further medium was added during culture, was very beneficial for the growth of the culture, it turned out to be less effective for the production of polyphenolic compounds: the levels of most of the analyzed metabolites, including all phenylethanoids was lower. Also, lower biosynthesis of most bioactive compounds was observed when the frequency of immersion was reduced.

Ultimately, the highest levels of all phenylethanoids and most polyphenolic acids, including RA, were observed in the three-week batch culture (Table 1). In this protocol, the RA content $(16.6 \mathrm{mg} / \mathrm{g} \mathrm{DW})$ was over $20 \%$ higher than in shoots grown for two weeks, and over $40 \%$ higher than in shoots from the four-week-old fed-batch culture. In the three-week batch system, the highest content, or close to it, was achieved by all polyphenolic acids, and thus the greatest 

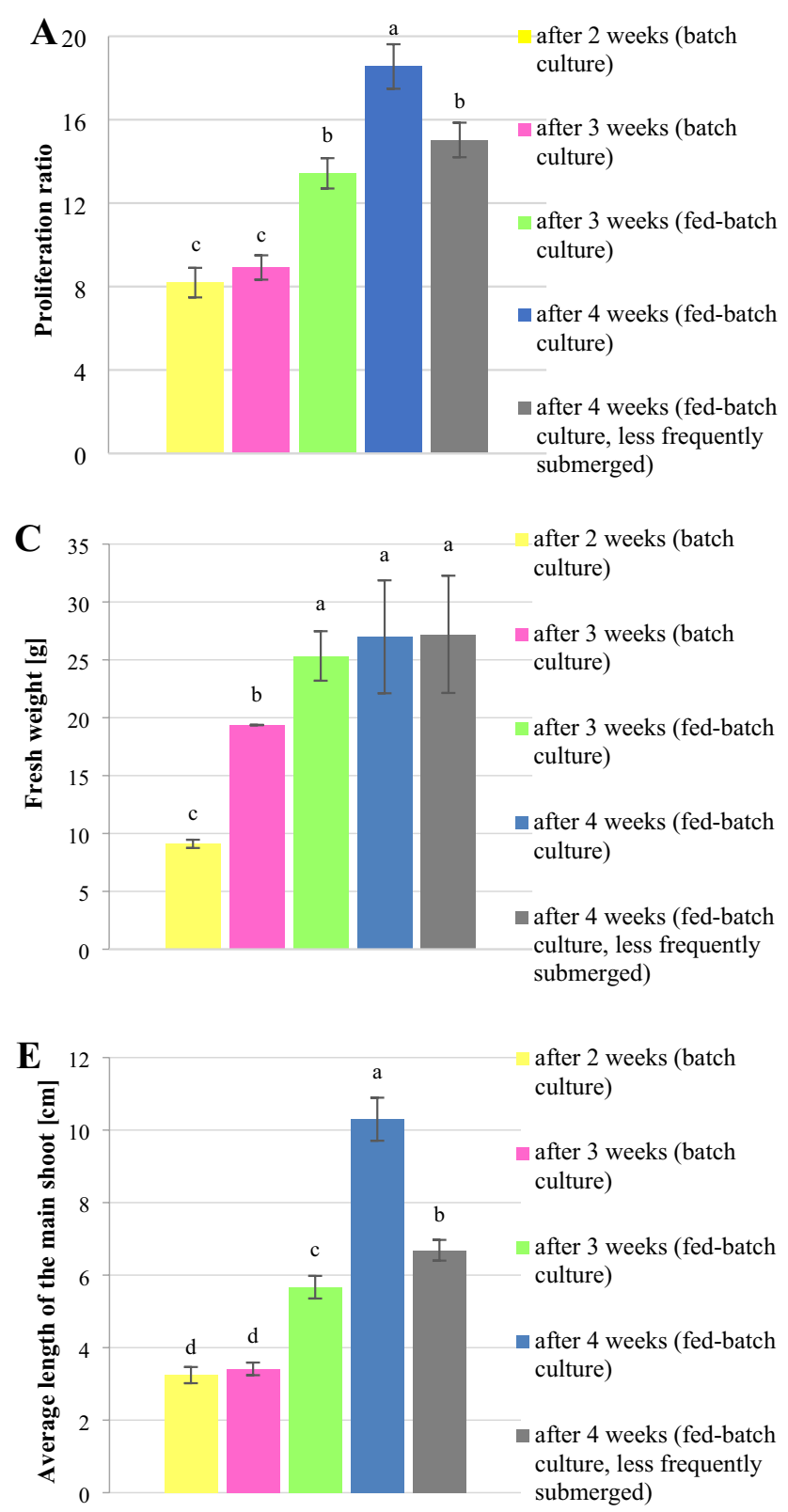

Fig. 2 Effect of different cultivation variants of Salvia viridis L. shoots in the Plantform bioreactor on: A proliferation ratio, $\mathbf{B}$ ratio of shoots to buds, $\mathbf{C}$ fresh weight, $\mathbf{D}$ dry weight, $\mathbf{E}$ length of the main shoot, and $\mathbf{F}$ length of axillary shoots. The results presented are mean

total polyphenolic acid content was reported (Table 1). However, the levels of other polyphenolic acid derivatives identified in the tested material were significantly lower than RA content; apart from methyl rosmarinate, they did not exceed $0.5 \mathrm{mg} / \mathrm{g}$ DW.

The three-week batch culture was also the most favourable for the biosynthesis of all phenylethanoids. The verbascoside (VB) level achieved was $9.80 \mathrm{mg} / \mathrm{g}$ DW. The conversion of the batch cultivation system to the fed-batch system reduced the VB content in the shoots by almost half, but
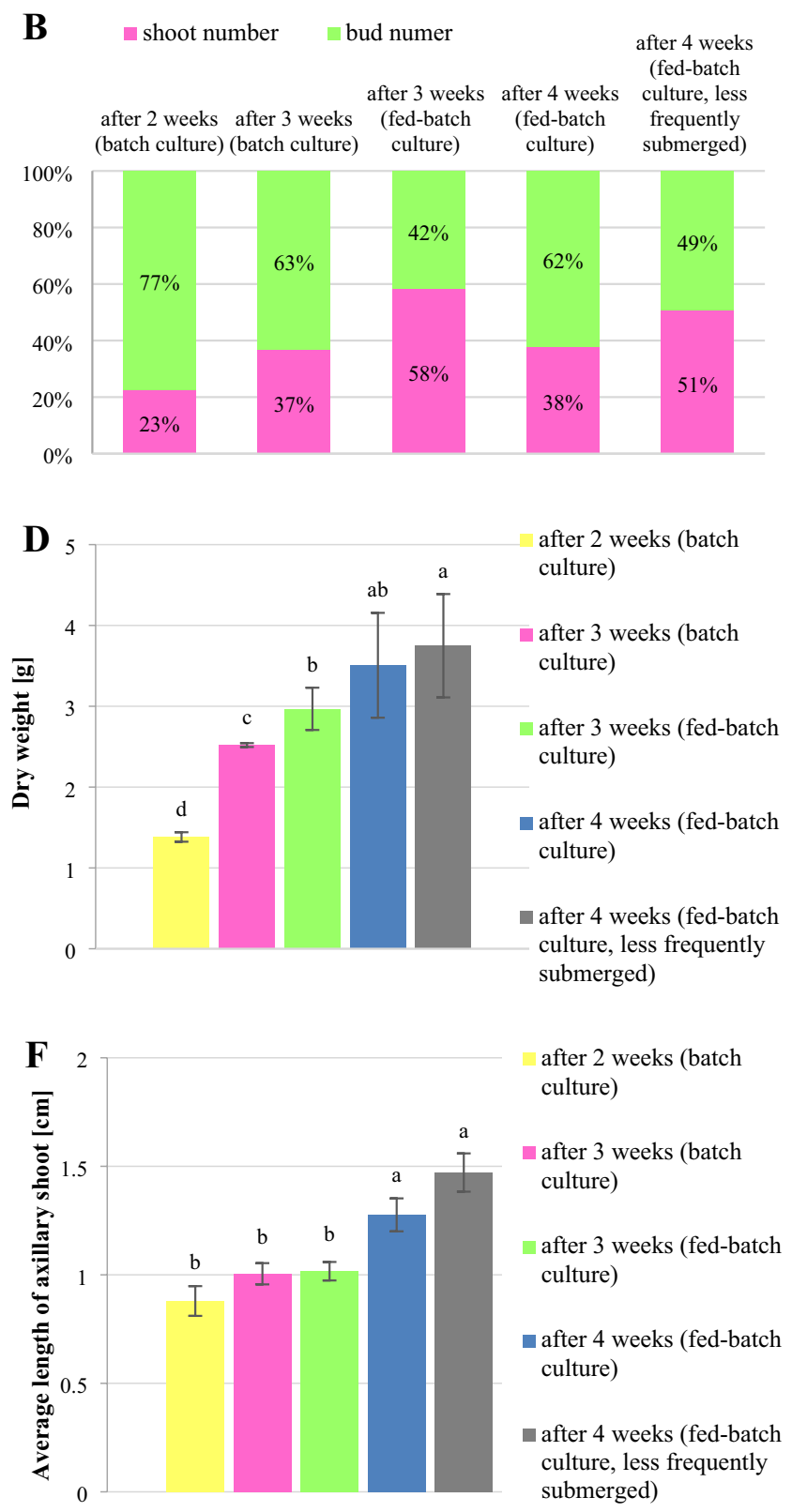

values \pm standard error (SE). Results were compared with the Tukey test at 5\% statistical significance. Values marked with the same letter were not significantly different (at $p \leq 0.05$ )

extending the duration of the fed-batch culture by another week increased production, and the final level was only $11 \%$ lower than observed for the three-week batch culture, which gave optimal phenylethanoid production (Table 1). The three-week cultivation variant also proved to be the most effective for producing the other tested phenylethanoids. The second most abundant compound in this group was LEU, the level of which exceeded $1 \mathrm{mg} / \mathrm{g}$ DW for the optimal cultivation variant. The three-week batch system thus ultimately proved to be the most efficient in terms of phenolic 


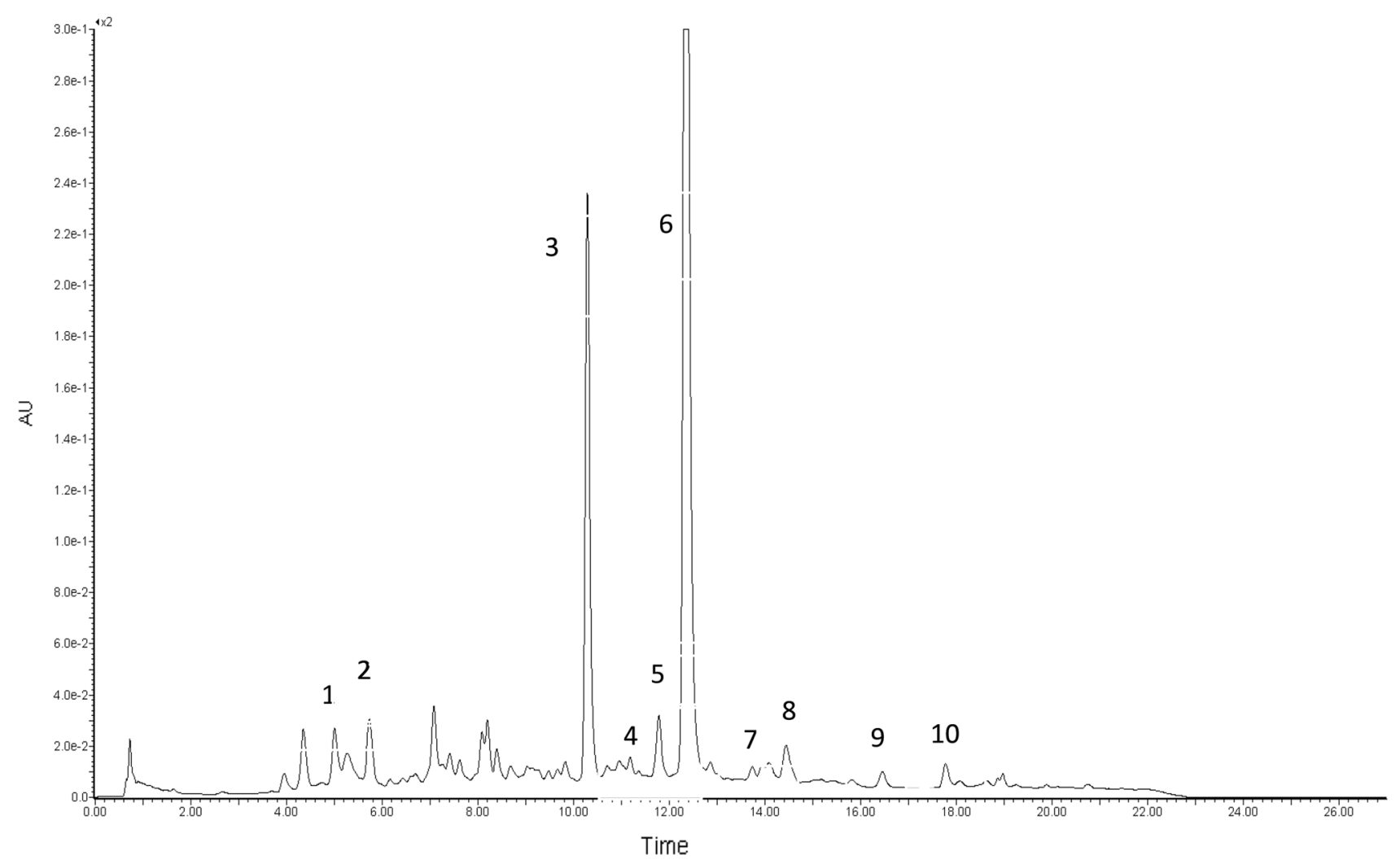

Fig. 3 Sample HPLC chromatogram of the hydromethanolic extract from Plantform bioreactor-cultivated shoot culture of S. viridis. 1-CAH, 2-CA, 3-VB, 4-ISOVB, 5-LEU, 6-RA, 7-MART, 8-MRA, 9-SAF(I), 10-SAF(II)

compound production, followed by the four-week fed-batch culture system (Table 1).

Since the most efficient for growth cultivation variant did not turn out to be optimal for the production of bioactive metabolites, it was therefore necessary to calculate the metabolite production in the biomass obtained for the different treatments during a single bioreactor cycle (Fig. 4). The highest total phenol production in $S$. viridis shoots during a single culture cycle was observed for the four-week fedbatch system $(80.8 \mathrm{mg})$ followed by the three-week batch culture $(74.8 \mathrm{mg})$; however, the differences were not statistically significant. Similar observations were made for total phenolic acid and phenylethanoid content; although in the case of the latter group, the difference between the two treatments was statistically significant, being approximately $20 \%$ higher than the three-week batch culture. On the other hand, some individual metabolites demonstrated optimal production in the three-week batch system. Meanwhile, similar production of RA, the predominant metabolite in the cultured in vitro sage shoots, was observed for the three-week batch system and four-week fed-batch cultures, at both the higher (every $80 \mathrm{~min}$ ) and lower (every 170) immersion frequencies: $41.7 \mathrm{mg}, 40.6 \mathrm{mg}$ and $37.6 \mathrm{mg}$, respectively (no statistical differences) (Fig. 4).

\section{Discussion}

In recent years, numerous studies have been conducted to assess the potential of natural raw materials and the possibility of their use in the prevention and treatment of various diseases. One example of such a species is Salvia viridis, a plant used in traditional medicine in Turkey and Iran, and one confirmed to have pharmacological potential. To harness the potential of a locally-occurring species on a large scale, it is necessary to identify methods that will provide unlimited access to standardized material, rich in bioactive compounds, regardless of its natural occurrence. In addition, to be effective and economically profitable, the production of plant material must be carried out on a large scale; such approaches are used for fungal or bacterial metabolites, where production is often carried out on an industrial scale in bioreactors with volumes of several thousand litres. However, changing the cultivation of plant organs from solid to liquid media is much more difficult (GrzegorczykKarolak et al. 2021). It requires not only upscaling: the use of larger culture vessels with higher volumes of medium, it also entails other technical issues, such as the sensitivity of plant tissues to the shear forces occurring during mixing or the limited access to gases when the explants are completely 
Table 1 Contents of phenolic compounds [mg/g DW] in Salvia viridis L. shoots cultured in the Plantform bioreactor, in MS liquid medium with $0.1 \mathrm{mg} / \mathrm{L} \mathrm{IAA}$ and $1 \mathrm{mg} / \mathrm{L} \mathrm{m}$-Top under different cultivation conditions

\begin{tabular}{|c|c|c|c|c|c|}
\hline \multirow[t]{2}{*}{ Compound } & \multicolumn{5}{|c|}{ Content of phenolic compounds [mg/g DW] } \\
\hline & $\begin{array}{l}\text { After } 2 \text { weeks } \\
\text { (batch culture) }\end{array}$ & $\begin{array}{l}\text { After } 3 \text { weeks } \\
\text { (batch culture) }\end{array}$ & $\begin{array}{l}\text { After } 3 \text { weeks (fed- } \\
\text { batch culture) }\end{array}$ & $\begin{array}{l}\text { After } 4 \text { weeks (fed- } \\
\text { batch culture) }\end{array}$ & $\begin{array}{l}\text { After } 4 \text { weeks (fed-batch } \\
\text { culture, less frequently sub- } \\
\text { merged) }\end{array}$ \\
\hline \multicolumn{6}{|l|}{ Phenolic acids } \\
\hline Rosmarinic acid & $13.40 \pm 0.21 b$ & $16.57 \pm 0.68 \mathrm{a}$ & $6.86 \pm 0.15 \mathrm{e}$ & $11.57 \pm 0.21 \mathrm{c}$ & $10.03 \pm 0.16 \mathrm{~d}$ \\
\hline Methyl rosmarinate & $0.56 \pm 0.006 \mathrm{a}$ & $0.49 \pm 0.01 b$ & $0.50 \pm 0.01 b$ & $0.42 \pm 0.004 \mathrm{c}$ & $0.41 \pm 0.004 \mathrm{c}$ \\
\hline Caffeic acid hexoside & $0.13 \pm 0.005 \mathrm{~d}$ & $0.25 \pm 0.01 b$ & $0.17 \pm 0.006 \mathrm{c}$ & $0.49 \pm 0.04 \mathrm{a}$ & $0.18 \pm 0.04 \mathrm{c}$ \\
\hline Caffeic acid & $0.19 \pm 0.004 b$ & $0.24 \pm 0.008 \mathrm{a}$ & $0.18 \pm 0.005 b$ & $0.10 \pm 0.03 c$ & $0.09 \pm 0.05 c$ \\
\hline Salvianolic acid F (I) & $0.31 \pm 0.01 \mathrm{a}$ & $0.33 \pm 0.009 a$ & $0.33 \pm 0.04 \mathrm{a}$ & $0.35 \pm 0.01 \mathrm{a}$ & $0.16 \pm 0.01 b$ \\
\hline Salvianolic acid F (II) & $0.24 \pm 0.01 \mathrm{c}$ & $0.39 \pm 0.01 b$ & $0.45 \pm 0.01 \mathrm{a}$ & $0.48 \pm 0.02 \mathrm{a}$ & $0.16 \pm 0.02 \mathrm{~d}$ \\
\hline \multicolumn{6}{|l|}{ Phenylethanoids } \\
\hline Verbascoside & $3.47 \pm 0.08 \mathrm{~d}$ & $9.80 \pm 0.43 \mathrm{a}$ & $5.10 \pm 0.12 \mathrm{c}$ & $8.68 \pm 0.22 b$ & $5.50 \pm 0.08 \mathrm{c}$ \\
\hline Leucosceptoside A & $0.75 \pm 0.02 b$ & $1.13 \pm 0.05 \mathrm{a}$ & $0.55 \pm 0.022 \mathrm{c}$ & $0.72 \pm 0.017 b$ & $0.52 \pm 0.009 \mathrm{c}$ \\
\hline Isoverbascoside & $0.17 \pm 0.01 \mathrm{c}$ & $0.33 \pm 0.03 \mathrm{a}$ & $0.15 \pm 0.02 \mathrm{c}$ & $0.13 \pm 0.03 c$ & $0.26 \pm 0.02 b$ \\
\hline Martynoside & $0.07 \pm 0.05 b$ & $0.16 \pm 0.02 \mathrm{a}$ & $0.05 \pm 0.04 \mathrm{c}$ & $0.07 \pm 0.01 b c$ & $0.05 \pm 0.01 \mathrm{c}$ \\
\hline \multicolumn{6}{|l|}{ Total compound content } \\
\hline Phenolic acid & $14.8 \pm 0.25 b$ & $18.3 \pm 0.73 a$ & $8.5 \pm 0.19 \mathrm{~d}$ & $13.4 \pm 0.25 b$ & $11.0 \pm 0.20 \mathrm{c}$ \\
\hline Phenylethanoid & $4.5 \pm 0.11 \mathrm{~d}$ & $11.4 \pm 0.54 \mathrm{a}$ & $5.8 \pm 0.17 \mathrm{c}$ & $9.6 \pm 0.25 b$ & $6.3 \pm 0.12 \mathrm{c}$ \\
\hline Phenol & $19.3 \pm 0.36 \mathrm{c}$ & $29.7 \pm 1.27 \mathrm{a}$ & $14.3 \pm 0.36 \mathrm{e}$ & $23.0 \pm 0.5 b$ & $17.3 \pm 0.32 \mathrm{~d}$ \\
\hline
\end{tabular}

The results presented are mean values \pm standard error (SE) of nine replicates for each plant material

Results were compared with Tukey's test at $5 \%$ statistical significance

Values marked with the same letter were not significantly different (at $\mathrm{p} \leq 0.05$ )

Fig. 4 The effects of different cultivation variants for Salvia viridis L. shoots in the Plantform bioreactor on total phenolic acid (TPA), phenylethanoid (TPE) and polyphenol (TP) content. The results presented are mean values \pm standard error (SE) of nine replicates for each plant material. Results were compared with Tukey's test at $5 \%$ statistical significance. Values marked with the same letter were not significantly different (at $\mathrm{p} \leq 0.05$ )

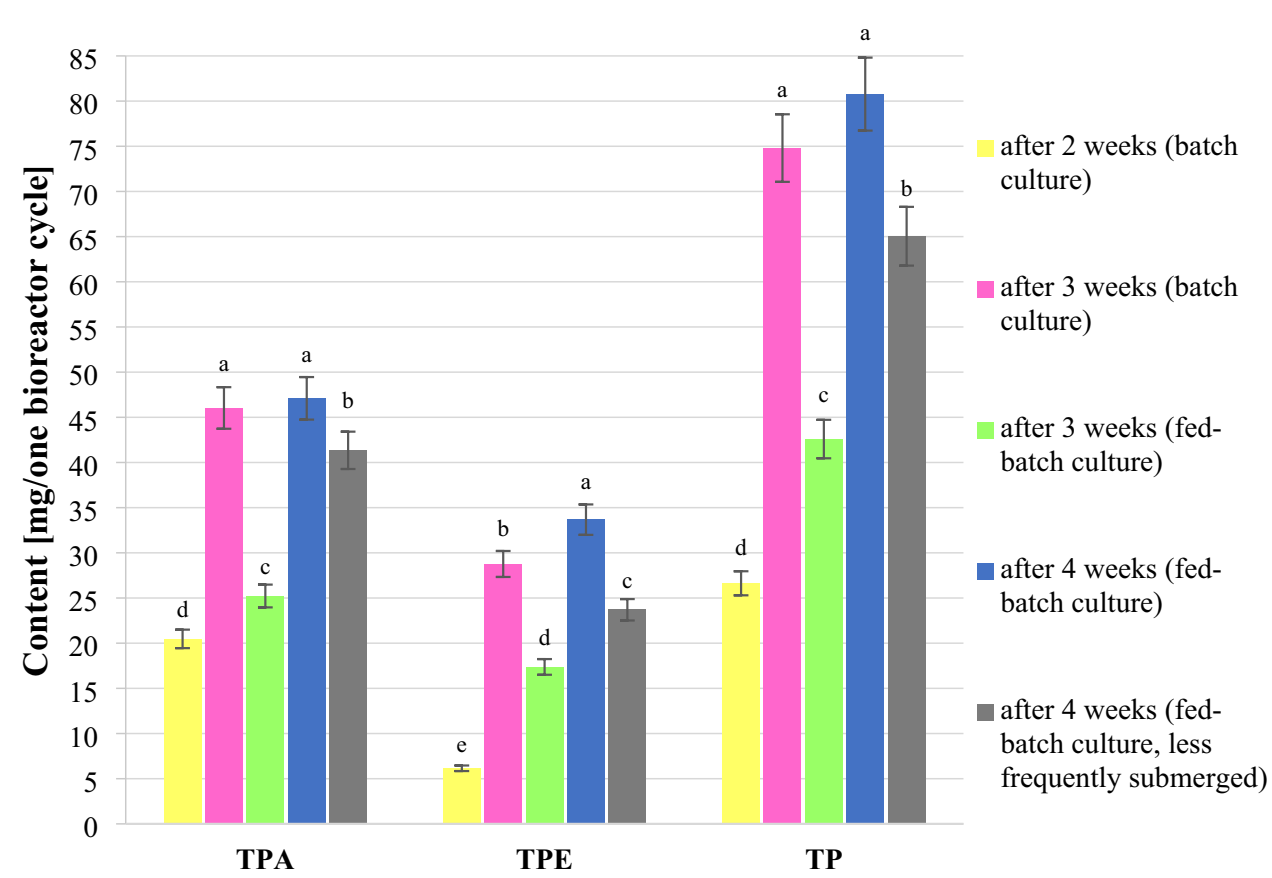

The present study demonstrates the influence of various cultivation conditions on the growth and the production of bioactive metabolites in $S$. viridis shoots cultured in the 
Plantform bioreactor. No data currently exists on the largescale in vitro cultivation of this species, nor on the use of Plantform bioreactors for the cultivation of other species of Salvia. This bioreactor type has previously demonstrated promising results when cultivating plant tissues in temporary immersion systems and has been successfully used for other medicinal species, such as Echinacea purpurea, Rubus idaeus (Welander et al. 2014) or Schisandra chinensis (Szopa et al. 2017). Temporary immersion systems (TISs) such as the Plantform system are characterized by extremely good tolerance for plants, they are cheap and easy to use, and they can be upscaled to industrial levels thanks to the possibility of serial connection. Such a solution could be useful for the effective production of large biomasses, which can produce large amounts of bioactive secondary metabolites.

However, like others, this system requires optimization for a given species. Various factors can influence the multiplication efficiency and production of secondary metabolites in shoot cultures grown in TISs. Two key parameters are the immersion frequency of plant material in the medium and the length of contact with the medium during the cycle. These factors influence the amount of nutrients delivered and the humidity inside the culture vessel, and can trigger stress associated with complete immersion in the medium; this can affect the amount of biomass obtained and the proliferation ratio, as well as the quality of the plant material obtained, its hydration level and vitreousness (Teisson et al. 1996).

Pérez-Alonso et al. (2009) report that the frequency of immersion in the medium (two minutes of immersion, every $2,4,6$ or $12 \mathrm{~h}$ ) significantly influences the biomass of Digitalis purpurea $\mathrm{L}$. shoot culture, its degree of hydration and proliferation ratio; however, immersion frequency did not appear to have any significant effect on shoot length. In the case of the $S$. viridis shoot cultures grown in the Plantform bioreactor, no significant differences in culture biomass were found between the two immersion frequencies (every 80 and $170 \mathrm{~min}$ ). Despite this, it was found that $S$. viridis shoots immersed more frequently in the medium demonstrated a higher proliferation ratio (18.6) than those immersed less frequently, despite being cultivated for the same amount of time. The plant material which had less frequent contact with the medium was characterised by shorter main shoots (approximately 1.5-fold), but slightly longer axillary shoots (1.2-fold).

The differences observed in response to frequency of immersion between Salvia viridis and Digitalis purpurea L. cultures may be specific to the species, but could also be related to the duration of submergence in the growth medium: in the present study, $S$. viridis culture was submerged for five times longer (10 min) than described by Pérez-Alonso et al. for Digitalis purpurea. In other studies, out of two immersion frequencies, viz. $15 \mathrm{~min} / 4 \mathrm{~h}$ and
$15 \mathrm{~min} / 8 \mathrm{~h}$, the latter was found to be more favourable for growth of Myrtus communis L. (Aka Kaçar et al. 2020). Similarly, immersion every eight hours was found to be optimal for the multiplication of Stevia rebaudiana Bert. (RamírezMosqueda et al. 2016). In contrast, however, changing the frequency of immersion (i.e. every 4,8 and $12 \mathrm{~h}$ ) did not result in any differences in biomass or multiplication for Anthurium andreanum Lind. (Martínez-Estrada et al. 2019).

Another factor influencing the amount of plant material obtained and the production level of secondary metabolites is the duration of culture. Prolonging the culture of Schisandra chinensis shoots in RITA $®$ and Plantform bioreactors has been found to result in the biomass and proliferation ratio more than doubling; however, this resulted in a lower production of lignans, which may indicate a depletion of nutrients in the supplied medium during the experiment (Szopa et al. 2017). In the present study, prolonging the duration of culture also proved to be more beneficial for the growth of $S$. viridis shoots in the Plantform bioreactor. From two to three weeks of culture (at the same immersion frequency) a twofold increase in FW and DW was observed, together with elongation of the main and axillary shoots, although the proliferation ratio increased only slightly (from 8.2 to 8.9). However, the ratio of shoots to buds increased from 23 to $37 \%$, suggesting that this procedure may make it possible to obtain more material capable of subsequent micropropagation without the need to further stimulate bud elongation. Longer culture durations were associated with adverse morphological changes, such as intense darkening of the lower parts of the shoots, and shoot necrosis. Similar effects were observed for S. chinensis (Szopa et al. 2017); the authors attribute this to the decrease in nutrient concentration in the medium or the accumulation of toxic metabolites inhibiting shoot growth. Therefore, the present study also examined the effect of adding a fresh portion of medium during the culture.

Some literature data indicate the possibility of using a semi-continuous system (fed-batch) to optimise plant culture growth and the biosynthesis of bioactive metabolites. The fed-batch system was compared with the batch system by Ritala et al. (2014), who cultivated the transformed tobacco (Nicotiana tabacum L.) roots in a bioreactor. Briefly, $20 \%$ of the initial volume of fresh medium was added to the culture after 8,15 and 22 days. The procedure resulted in a 56\% increase of DW in comparison to the batch culture and 1.6 times higher geraniol biosynthesis in the obtained biomass. Similarly, in the present study, the addition of fresh medium increased the proliferation of $S$. viridis shoots in the Plantform bioreactor. After three weeks, the fed-batch culture demonstrated a $31 \%$ increase in $\mathrm{FW}, 18 \%$ increase in DW, a $50 \%$ increase in proliferation ratio, and a $66 \%$ increase in main shoot length compared to the batch culture. No significant differences were observed in the case of axillary shoot 
length. However, the shoot to bud ratio increased from 37 to $58 \%$. In addition, the duration of culture could be extended up to four weeks.

The growth dynamics of the $S$. viridis shoot culture in the Plantform bioreactor were significantly different to a similar culture performed on the solid agar medium of identical composition (MS with the addition of $0.1 \mathrm{mg} / \mathrm{l}$ IAA and $1 \mathrm{mg} / \mathrm{l} \mathrm{m}$-Top) (Grzegorczyk-Karolak et al. 2020). In the latter, the shoot growth cycle lasted 5 weeks, during which time, a 20 -fold increase in biomass was achieved; in contrast, a 33-fold increase in biomass was obtained in the semi-continuous bioreactor culture within four weeks. This more intensive increase of culture mass may be attributed to differences in shoot length: the main and axillary shoots grown in the bioreactor significantly longer. In addition, the proliferation ratio was four times higher. In conclusion, the $S$. viridis shoots cultured in liquid medium in the Plantform bioreactor demonstrate faster growth processes than those on solid medium, probably due to the better availability of nutrients contained in the medium. Immersion in the medium allowed the shoots to absorb substances necessary for growth with their whole surface, not only through the lower parts. Similar observations were made in an experiment in which Salvia officinalis L. shoots were grown in a liquid medium (Grzegorczyk and Wysokińska 2008); this culture demonstrated more intensive growth than that grown on agar medium of the same composition for two weeks longer. The shoots obtained in the conditions of a liquid medium produced more leaves, which reached larger sizes. While both cases demonstrated similar numbers of formed shoots, liquid culture demonstrated greater biomass and a considerably shorter cultivation time.

Another aim of the present study was to optimise the cultivation conditions of $S$. viridis shoots grown in the Plantform bioreactor to allow efficient biosynthesis of secondary metabolites. It was found that the production of bioactive compounds differed according to the choice of culture technique, its duration and immersion frequency, and that the conditions that were most favourable for culture growth were not optimal for secondary metabolite production. This may have been due to a significant change in the growth cycle and metabolism of the culture caused by the supplementation. The most efficient biosynthesis of phenolic acids and phenylethanoids, with total content of approximately $30 \mathrm{mg} / \mathrm{g} \mathrm{DW}$, was observed in the three-week batch culture, immersed every $80 \mathrm{~min}$ (Table 1 ). The two main secondary metabolites were rosmarinic acid, at a concentration of $16.6 \mathrm{mg} / \mathrm{g}$ DW, and verbascoside, at a concentration of $9.8 \mathrm{mg} / \mathrm{g}$ DW. The RA levels were twice those obtained in shoots cultivated on the solid medium, and 13 times more than in aerial parts of soil-grown plants (Grzegorczyk-Karolak and Kiss 2018; Grzegorczyk-Karolak et al. 2020). For VB, this value was similar to that of the soil-grown shoots and 1.8 times higher than the in vitro cultures grown on solid medium. It should also be noted that the material could be obtained more quickly by liquid culture: 5 weeks were needed for agar culture and 16 weeks for soil cultivation. Plantform bioreactor culture thus appears to be an exceptionally advantageous method of obtaining biologically-active compounds from Salvia viridis; in addition, unlike traditional field cultivation, it offers the possibility of continuous, reproducible continuation of cultivation, regardless of climate or season.

The potential for bioreactor cultivation to deliver larger amounts of secondary metabolites in a shorter time has also been noted in previous studies. For example, nutrient sprinkle bioreactor culture of Salvia officinalis L. shoots yielded $26.2 \mathrm{mg} / \mathrm{g}$ DW of rosmarinic acid after three weeks; this value was more than twice that obtained for 10 -week-old shoots grown in soil, i.e. $12.2 \mathrm{mg} / \mathrm{g}$ DW (Grzegorczyk and Wysokińska, 2010). Ruta et al. (2020) also note that the total phenolic content (calculated as gallic acid) in Lycium barbarum L. shoots cultivated in the Plantform bioreactor was more than twice $(23.6 \mathrm{mg} / \mathrm{g} \mathrm{DW})$ that found in shoots grown in the natural environment (11.98 mg/g DW). Previous studies also suggest that shoot cultures grown in bioreactors with a temporary immersion system offer promising potential for efficient production of a variety of secondary metabolites with therapeutic properties: lignan biosynthesis in Schisandra chinensis (Szopa et al. 2017), cardenolide glycosides in Digitalis purpurea (Perez-Alonso et al. 2009) or volatile compounds in Rhododendron tomentosum Harmaja (Jesionek et al. 2017).

Finally, it should be remembered that several factors influence the effectiveness of the culture, not only the final content of compounds in the obtained plant material and the increase in biomass, but also the duration of the culture and the cost of preparation and supplementation of the medium. The three-week batch culture and four-week fed-batch system offered comparable efficiency in the production of bioactive compounds in S. viridis shoot culture (Fig. 4). In fact the former appears more efficient and cost effective: despite weaker culture growth and proliferation, the obtained plant material was richer in bioactive secondary metabolites; in addition, culture time was shorter and did not require further medium supplementation. There was also no chance of culture contamination associated with the addition of the medium. Ultimately, within three weeks, $46 \mathrm{mg}$ of phenolic acids were obtained, including $41.7 \mathrm{mg}$ of RA and $28.8 \mathrm{mg}$ of phenylethanoids, including $24.7 \mathrm{mg}$ of VB.

\section{Conclusions}

The presented findings indicate for the first time that effective cultivation of Salvia viridis shoots in the Plantform bioreactor with a temporary immersion system is possible. 
The duration and technique of culture (batch or fed-batch) and the frequency of immersion in the liquid medium were shown to affect the biomass, proliferation ratio and the production of secondary metabolites. The obtained results are innovative and have practical applications, because culture obtained large amounts of plant material rich in the bioactive compounds. The most favourable growth parameters were achieved for plants grown for 4 weeks in a semi-continuous (fed-batch) system, in which $26.99 \mathrm{~g} \mathrm{FW}$ and $3.51 \mathrm{~g} \mathrm{DW}$ of shoots were obtained, with a proliferation ratio of 18.6. However, ultimately, the highest production of bioactive phenolic compounds was found in the 3 -week batch culture: the obtained total phenolic acid content was about 10 times higher than in plants grown in soil for four months and more than two times higher than in shoot culture grown for 5 weeks on solid medium. In addition, after three-week batch culture in the Plantform bioreactor, the total phenylethanoid level in S. viridis shoots was $40 \%$ higher than that found in shoots from five-week solid culture and similar to that in the aerial parts of plants grown in the soil for 16 weeks. These figures demonstrate the potential of such culture methods, both in industry, for producing medically important compounds, and in research, for obtaining large amounts of valuable biomass for further research.

Author Contribution IG-K conception and design of the study, PS and IG-K conducting biotechnological experiments, LL performing HPLC analysis, PS and IG-K analysis and interpretation of data, DGP interpretation of phytochemical results, PS writing original draft of the manuscript, IG-K statistical analysis, review and editing the manuscript. All authors read and approved the manuscript.

Funding This research was supported by Medical University of Lodz, a Grant No. 503/3-012-01/503-31-001-19-00.

Data Availability The data that support the findings of this study are available from the corresponding author on reasonable request.

\section{Declarations}

Conflict of interest The authors declare no conflict of interest.

Open Access This article is licensed under a Creative Commons Attribution 4.0 International License, which permits use, sharing, adaptation, distribution and reproduction in any medium or format, as long as you give appropriate credit to the original author(s) and the source, provide a link to the Creative Commons licence, and indicate if changes were made. The images or other third party material in this article are included in the article's Creative Commons licence, unless indicated otherwise in a credit line to the material. If material is not included in the article's Creative Commons licence and your intended use is not permitted by statutory regulation or exceeds the permitted use, you will need to obtain permission directly from the copyright holder. To view a copy of this licence, visit http://creativecommons.org/licenses/by/4.0/.

\section{References}

Aka Kaçar Y, Biçen B, Şimşek Ö, Dönmez D, Erol MH (2020) Evaluation and comparison of a new type of temporary immersion system (TIS) bioreactors for myrtle (Myrtus communis L.). Appl Ecol Environ Res 18(1):1611-1620

Alipieva K, Korkina L, Orhan IE, Georgiev MI (2014) Verbascosidea review of its occurrence, (bio)synthesis and pharmacological significance. Biotechnol Adv 32(6):1065-1076

Almusawi AHA, Sayegh AJ, Alshanaw AMS, Griffis JL Jr (2017) Plantform bioreactor for mass micropropagation of date palm. Methods Mol Biol 1637:251-265

Astani A, Navid MH, Schnitzler P (2014) Attachment and penetration of acyclovir-resistant herpes simplex virus are inhibited by Melissa officinalis extract. Phytother Res 28(10):1547-1552

Benelli C, De Carlo A (2018) In vitro multiplication and growth improvement of Olea europaea L. cv Canino with temporary immersion system (Plantform). 3 Biotech 8(7):317

Bown D (2002) The Royal Horticultural Society New Encyclopedia of Herbs \& Their Uses. Dorling Kindersley Publishers, London, pp 353-356

Damiano C, La Starza SR, Monticelli S, Gentile A, Caboni E, Frattarelli A (2005) Propagation of Prunus and Malus by temporary immersion. In: Hvoslef-Eide AK, Preil W (eds) Liquid culture systems for in vitro plant propagation. Springer, Netherlands Dordrecht

Davis PH (1982) Flora of Turkey and the East Aegean Islands. Edinburgh University Press, Edinburgh

Dweck AC (2000) The folklore and cosmetic use of various Salvia species. In: Spiridon E. Kintzios (ed) Sage: the genus Salvia, 14th edn., Harwood Academic Publishers The Netherlands.

Elufioye TO, Habtemariam S (2019) Hepatoprotective effects of rosmarinic acid insight into its mechanisms of action. Biomed Pharmacother 112:108600

Espinosa-Leal CA, Puente-Garza CA, García-Lara S (2018) In vitro plant tissue culture: means for production of biological active compounds. Planta 248:1-18

Ghorbani A (2005) Studies on pharmaceutical ethnobotany in the region of Turkmen Sahara, north of Iran: (Part 1): general results. J Ethnopharmacol 102:58-68

Grzegorczyk I, Wysokińska H (2008) Liquid shoot culture of Salvia officinalis $\mathrm{L}$. for micropropagation and production of antioxidant compounds; effect of triacontanol. Acta Soc Bot Pol 77(2):99-104

Grzegorczyk I, Wysokińska H (2010) Antioxidant compounds in Salvia officinalis L. shoot and hairy root cultures in the nutrient sprinkle bioreactor. Acta Soc Bot Pol 79(1):7-10

Grzegorczyk-Karolak I (2020) Optimization of culture conditions and cultivation phase for the growth of Salvia viridis transformed roots and polyphenolic compound production. Plant Cell Tissue Organ Cult 142:571-581

Grzegorczyk-Karolak I, Grąbkowska R, Piątczak E (2021) Plant liquid cultures as a source of bioactive metabolites. In: Ramawat KG, Ekiert HM, Goyal S (eds) Plant cell and tissue differentiation and secondary metabolites. Reference Series in Phytochemistry, Springer Cham, pp 743-771

Grzegorczyk-Karolak I, Hnatuszko-Konka K, Zarzycka M, Kuźma Ł (2020) The stimulatory effect of purine-type cytokinins on proliferation and polyphenolic compound accumulation in shoot culture of Salvia viridis. Biomolecules 10(2):178

Grzegorczyk-Karolak I, Kiss AK (2018) Determination of the phenolic profile and antioxidant properties of Salvia viridis L. shoots: a comparison of aqueous and hydroethanolic extracts. Molecules 23(6): 1468

Grzegorczyk-Karolak I, Kuźma Ł, Lisiecki P, Kiss AK (2019) Accumulation of phenolic compounds in different in vitro cultures of 
Salvia viridis $\mathrm{L}$. and their antioxidant and antimicrobial potential. Phytochem Lett 30:324-332

Grzegorczyk-Karolak I, Kuźma Ł, Skała E, Kiss AK (2018) Hairy root cultures of Salvia viridis L. for production of polyphenolic compounds. Ind Crops Prod 117:235-244

Habtemariam S (2018) Molecular pharmacology of rosmarinic and salvianolic acids: potential seeds for Alzheimer's and vascular dementia drugs. Int J Mol Sci 19(2):458

Hu XP, Shao MM, Song X, Wu XL, Qi L, Zheng K, Fan L, Liao CH, Li CY, He J, Hu YJ, Wu HQ, Li SH, Zhang J, Zhang FX, He ZD (2016) Anti-influenza virus effects of crude phenylethanoid glycosides isolated from Ligustrum purpurascens via inducing endogenous interferon- $\gamma$. J Ethnopharmacol 179:128-136

Jesionek A, Kokotkiewicz A, Włodarska P, Zabiegała B, Buciński A, Luczkiewicz M (2017) Bioreactor shoot cultures of Rhododendron tomentosum (Ledum palustre) for a large-scale production of bioactive volatile compounds. Plant Cell Tissue Organ Cult 131(1):51-64

Lee W, Yang EJ, Ku SK, Song KS, Bae JS (2013) Anti-inflammatory effects of oleanolic acid on LPS-induced inflammation in vitro and in vivo. Inflammation 36(1):94-102

Liang JQ, Wang L, He JC, Hua XD (2016) Verbascoside promotes the regeneration of tyrosine hydroxylase-immunoreactive neurons in the substantia nigra. Neural Regen Res 11(1):101-106

López-Rodríguez R, Herrera-Ruiz M, Trejo-Tapia G, Domínguez-Mendoza BE, González-Cortazar M, Zamilpa A (2019) In vivo gastroprotective and antidepressant effects of iridoids, verbascoside and tenuifloroside from Castilleja tenuiflora Benth. Molecules 24(7):1292

Luo C, Zou L, Sun H, Peng J, Gao C, Bao L, Ji R, Jin Y, Sun S (2020) A review of the anti-inflammatory effects of rosmarinic acid on inflammatory diseases. Front Pharmacol 11:153

Lv R, Du L, Liu X, Zhou F, Zhang Z, Zhang L (2019) Rosmarinic acid attenuates inflammatory responses through inhibiting HMGB1/ TLR4/NF- $\mathrm{KB}$ signaling pathway in a mouse model of Parkinson's disease. Life Sci 223:158-165

Martínez-Estrada E, Islas-Luna B, Pérez-Sato JA, Bello-Bello JJ (2019) Temporary immersion improves in vitro multiplication and acclimatization of Anthurium andreanum Lind. Sci Hortic 249:185-191

Murashige T, Skoog F (1962) A revised medium for rapid growth and bio assays with tobacco tissue cultures. Physiol Plant 15(3):473-497

Nadeem M, Imran M, Aslam Gondal T, Imran A, Shahbaz M, Muhammad Amir R, Wasim Sajid M, Batool Qaisrani T, Atif M, Hussain G, Salehi B, Adrian Ostrander E, Martorell M, Sharifi-Rad J, Cho WC, Martins N (2019) Therapeutic potential of rosmarinic acid: a comprehensive review. Appl Sci 9:3139

Naimi M, Vlavcheski F, Shamshoum H, Tsiani E (2017) Rosemary extract as a potential anti-hyperglycemic agent: current evidence and future perspectives. Nutrients 9(9):968

Perez-Alonso N, Wilken D, Gerth A, Jahn A, Nitzsche HM, Kerns G, Capote-Perez A, Jimenez E (2009) Cardiotonic glycosides from biomass of Digitalis purpurea L. cultured in temporary immersion systems. Plant Cell Tissue Organ Cult 99:151-156

Quan W, Liu HX, Zhang W, Lou WJ, Gong YZ, Yuan C, Shao Q, Wang N, Guo C, Liu F (2021) Cardioprotective effect of rosmarinic acid against myocardial ischaemia/reperfusion injury via suppression of the NF- $\mathrm{\kappa B}$ inflammatory signalling pathway and ROS production in mice. Pharm Biol 59(1):222-231

Ramírez-Mosqueda MA, Iglesias-Andreu LG, Ramírez-Madero G, Hernández-Rincón EU (2016) Micropropagation of Stevia rebaudiana Bert. in temporary immersion systems and evaluation of genetic fidelity. S Afr J Bot 106:238-243
Ritala A, Dong L, Imseng N, Seppänen-Laakso T, Vasilev N, van der Krol S, Rischer H, Maaheimo H, Virkki A, Brändli J, Schillberg S, Eibl R, Bouwmeester H, Oksman-Caldentey KM (2014) Evaluation of tobacco (Nicotiana tabacum L. cv. Petit Havana SR1) hairy roots for the production of geraniol, the first committed step in terpenoid indole alkaloid pathway. J Biotechnol 176:20-28

Rungsimakan S, Rowan MG (2014) Terpenoids, flavonoids and caffeic acid derivatives from Salvia viridis L. cvar. Blue Jeans Phytochemistry 108:177-188

Ruta C, De Mastro G, Ancona S, Tagarelli A, De Cillis F, Benelli C, Lambardi M (2020) Large-scale plant production of Lycium barbarum L. by liquid culture in temporary immersion system and possible application to the synthesis of bioactive substance. Plants 9(7):844

Sharma GK, Jagetiya S, Dashora R (2015) General techniques of plant tissue culture. Lulu Press Inc, Raleigh, North Carolina, United States

Szopa A, Kokotkiewicz A, Luczkiewicz M, Ekiert H (2017) Schisandra lignans production regulated by different bioreactor type. J Biotechnol 247:11-17

Teisson C, Alvard D, Berthouly M, Cote F, Escalant J, Etienne H, Lartand M (1996) Simple apparatus to perform plant tissue culture by temporary immersion. Acta Hortic 440:521-526

Tewtrakul S, Miyashiro H, Nakamura N, Hattori M, Kawahata T, Otake T, Yoshinaga T, Fujiwara T, Supavita T, Yuenyongsawad S, Rattanasuwon P, Dej-Adisai S (2003) HIV-1 integrase inhibitory substances from Coleus parvifolius. Phytother Res 17(3):232-239

Tripathi L, Tripathi JN (2005) Role of biotechnology in medicinal plants. Trop J Pharm Res 2(2):243-253

Ulubelen A (2003) Cardioactive and antibacterial terpenoids from some Salvia species. Phytochemistry 64:395-399

Vieira LC, Moreira CPS, Castro BFM, Cotta OAL, Silva LM, Fulgêncio GO, Silva-Cunha A, Fialho SL (2020) Rosmarinic acid intravitreal implants: a new therapeutic approach for ocular neovascularization. Planta Med 17(86):1286-1297

Welander M, Persson J, Asp H, Zhu LH (2014) Evaluation of a new vessel system based on temporary immersion system for micropropagation. Sci Hortic 179:227-232

Welander M, Sayegh A, Hagwall F, Kuznetsova T, Holefors A (2017) Technical improvement of a new bioreactor for large scale micropropagation of several Vaccinium cultivars. Acta Hortic 1180:387-392

Wu L, Georgiev MI, Cao H, Nahar L, El-Seedi HR, Sarker SD, Xiao J, Lu B (2020) Therapeutic potential of phenylethanoid glycosides: a systematic review. Med Res Rev 40:2605-2649

Yayli N, Cansu TB, Yilmaz N, Yasar A, Cetin MM, Yayli N (2010) Constituents of the essential oil from the flower, leaf and stem of Salvia viridis L. grown in Turkey. Asian J Chem 22(5):3439-3446

Yun SY, Hur YG, Kang MA, Lee J, Ahn C, Won J (2003) Synergistic immunosuppressive effects of rosmarinic acid and rapamycin in vitro and in vivo. Transplantation 75(10): 1758-1760

Zengin G, Mahomoodally F, Picot-Allain C, Diuzheva A, Jekő J, Cziáky Z, Cvetanović A, Aktumsek A, Zeković Z, Rengasamy KRR (2019) Metabolomic profile of Salvia viridis L. root extracts using HPLC-MS/MS technique and their pharmacological properties: a comparative study. Ind Crops Prod 131:266-280

Publisher's Note Springer Nature remains neutral with regard to jurisdictional claims in published maps and institutional affiliations. 